\title{
VALIDADE DE CONSTRUTO DO ÍNDICE DE HUMANIZAÇÃO DOS SERVIÇOS DE SAÚDE*
}

\author{
Delvane José de Souza ${ }^{1}$, Lis Ribeiro de Oliveira ${ }^{2}$, Rejane Cussi Assunção Lemos ${ }^{3}$, Márcia Marques dos Santos \\ Felix $^{4}$, Maria D'Innocenzo ${ }^{5}$
}

RESUMO: Objetivou apresentar os resultados do estudo de validade de construto do Índice de Humanização dos Serviços de Saúde. Estudo metodológico de validação de indicadores de avaliação em saúde, desenvolvido em um complexo hospitalar e unidades de atenção primária e especializada, na cidade de Uberaba, estado de Minas Gerais. Participaram 312 trabalhadores, 211 usuários e 49 gestores, entre novembro de 2013 e março de 2014. As medidas de adequação da amostra indicaram ser possível uma solução fatorial. Foram nomeados 7 fatores na dimensão trabalho, 7 fatores na dimensão usuário e 3 fatores na dimensão gestão. $\mathrm{O}$ instrumento proposto atende aos requisitos psicométricos preconizados, podendo ser utilizado para avaliar a percepção de trabalhadores, usuários e gestores sobre aspectos da humanização dos serviços de saúde.

DESCRITORES: Análise fatorial; Estudos de validação; Humanização da assistência; Indicadores de serviços; Confiabilidade dos dados.

\section{CONSTRUCT VALIDITY OF THE HEALTH SERVICES HUMANIZATION INDEX}

\begin{abstract}
This study aimed to present the construct validity results of the Health Service Humanization Index. Methodological study to validate health assessment indicators, developed at a hospital complex and primary and specialized care services in the city of Uberaba, state of Minas Gerais. Participants were 312 workers, 211 users and 49 managers between November 2013 and March 2014. The sample adequacy measures indicated that a factorial solution is possible. Seven factors were indicated in the work dimension, seven in the user dimension and three in the management dimension. The proposed instrument complies with the recommended psychometric requisites and can be used to assess the perception of workers, users and managers about aspects of health service humanization.
\end{abstract}

DESCRIPTORS: Factor analysis; Validation studies; Care humanization; Service indicators; Data reliability.

\section{VALIDEZ DE CONSTRUCTO DEL ÍNDICE DE HUMANIZACIÓN DE LOS SERVICIOS DE SALUD}

RESUMEN: El objetivo fue presentar los resultados del estudio de validez de constructo del Índice de Humanización de los Servicios de Salud. Estudio metodológico de validación de indicadores de evaluación en salud, desarrollado en un complexo hospitalario y unidades de atención primaria y especializada en la ciudad de Uberaba, estado de Minas Gerais. Participaron 312 trabajadores, 211 usuarios y 49 gestores, entre noviembre del 2013 y marzo del 2014. Las medidas de adecuación de la muestra indicaron que una solución factorial es posible. Fueron nombrados 7 factores en la dimensión trabajo, 7 factores en la dimensión usuario y 3 factores en la dimensión gestión. El instrumento propuesto cumple con los requisitos psicométricos preconizados y puede ser utilizado para evaluar la percepción de trabajadores, usuarios y gestores sobre aspectos de la humanización de los servicios de salud.

DESCRIPTORES: Análisis factorial; Estudios de validación; Humanización de la atención; Indicadores de servicios; Confiabilidad de los datos.

\footnotetext{
*Artigo extraído da tese intitulada: "Avaliação da Implantação da Política Nacional de Humanização da Atenção e Gestão por meio da Construção do Índice de Humanização dos Serviços de Saúde". Universidade Federal de São Paulo, 2015.

${ }^{1}$ Enfermeiro. Doutor em Ciências. Enfermeiro do Curso de Graduação em Enfermagem da Universidade Federal do Triângulo Mineiro. Uberaba, MG, Brasil.

${ }^{2}$ Enfermeira. Mestre em Atenção à Saúde. Enfermeira da Prefeitura Municipal de Araxá. Araxá, MG, Brasil.

${ }^{3}$ Enfermeira. Mestre em Enfermagem. Docente de Enfermagem da Universidade Federal do Triângulo Mineiro. Uberaba, MG, Brasil.

${ }^{4}$ Enfermeira. Doutoranda em Atenção à Saúde. Universidade Federal do Triângulo Mineiro. Uberaba, MG, Brasil.

${ }^{5}$ Enfermeira. Docente do Programa de Pós-Graduação em Enfermagem da Universidade Federal de São Paulo. São Paulo, SP, Brasil.
}

Autor Correspondente:

Recebido: $11 / 01 / 2016$

Delvane José de Souza

Finalizado: 11/10/2016

Universidade Federal do Triângulo Mineiro

Pç. Manoel Terra, 330 - 38025-015 - Uberaba, MG, Brasil

E-mail: delvane.jose@gmail.com 


\section{- INTRODUÇÃO}

A Política Nacional de Humanização da Atenção e Gestão (PNH) levanta a necessidade de melhorar aspectos organizacionais dos serviços de saúde por meio de medidas humanizadoras, que resultam na mudança da cultura das práticas de atenção aos usuários e da gestão de processos de trabalho. Implica reconhecer muitos desafios, como a ampliação do acesso com qualidade aos serviços e aos bens de saúde, precarização de vínculos e modelos verticais de gestão. Prevê uma nova forma de operar, traduzindo em inovação na dinâmica organizacional, requisitando um aprofundamento na perspectiva avaliativa ${ }^{(1-3)}$.

A avaliação é parte do planejamento e gestão do sistema de saúde. A humanização, em virtude de sua complexidade, demanda a criação de indicadores capazes de dimensionar não somente o quadro de saúde e doença, mas as repercussões em outros níveis de representação dos sujeitos, inclusive as subjetivas. Por estar inserida nas políticas públicas e no sentido de dar visibilidade às prioridades e aplicação de recursos em cenários variados, a humanização deve ser colocada no contexto da

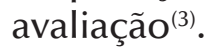

O desenvolvimento de pesquisas avaliativas fornece subsídios para o planejamento e estratégias de implantação das ações de saúde. Contribui para a elaboração de metodologias avaliativas de políticas públicas, em consonância com os princípios do Sistema Único de Saúde (SUS), bem como para o desenvolvimento de indicadores que podem apoiar práticas gerenciais voltadas para obter maior eficácia, qualidade e eficiência ${ }^{(3)}$.

O Índice de Humanização dos Serviços de Saúde (IHS) busca validar um instrumento de pesquisa quantitativo destinado a mensurar o grau de humanização dos serviços de saúde. Nesse sentido, "os critérios de validade de instrumento nas pesquisas científicas consistem em um elemento fundamental que compõe o rigor metodológico" (4:227). Estudos de validação são amplamente usados na pesquisa em diversas áreas do conhecimento na literatura mundial.

Um construto expressa, por meio de uma ou mais variáveis, o verdadeiro significado teórico de um conceito. "A validade de construto se refere ao grau em que um instrumento de medidas se relacione consistentemente com outras medições assemelhadas derivadas da mesma teoria e conceitos que estão sendo medidos" (5:7).

Considerando a importância das ações de humanização e a necessidade de avaliação da PNH enquanto política pública, esta pesquisa tem objetivo como apresentar os resultados do estudo de validade de construto do Índice de Humanização dos Serviços de Saúde (IHS).

\section{MÉTODOS}

Trata-se de um estudo quantitativo com abordagem metodológica. Os locais escolhidos foram um complexo hospitalar universitário com 290 leitos e um serviço municipal de atenção primária e ambulatorial especializado, da cidade de Uberaba, estado de Minas Gerais. No mês de outubro de 2013 esta rede de serviços contava com 1.545 trabalhadores em saúde e 72 gestores.

Foram utilizadas escalas ordinais tipo Likert com quatro opções de resposta, duas remetem a uma avaliação positiva e duas a uma avaliação negativa. As variáveis incluídas foram definidas por meio da técnica Delphi em uma etapa anterior da pesquisa. Para cada variável formulou-se um conjunto de afirmações capazes de estimar seu conteúdo. Seguiu-se o princípio de que o respondente expressasse sua opinião a respeito de condições relacionadas às diretrizes humanizantes presentes nos serviços de saúde pesquisados.

Os participantes foram selecionados pelo critério da amostra aleatória simples. Para o cálculo do tamanho da amostra foram utilizados dois critérios: o primeiro critério foi uma fórmula que atendesse aos critérios de variável qualitativa, população finita, nível de significância (95\%), erro amostral máximo tolerado $(5 \%)$, e desfecho erro $x$ acerto de $50 \%$; e o segundo critério o número mínimo de variáveis colocadas em votação. 
Os critérios de inclusão para os participantes da pesquisa foram: a) gestores e trabalhadores em saúde com vínculo empregatício na instituição sem tempo determinado; e b) usuários em atendimento no serviço, orientados do ponto de vista cognitivo e em condições clínicas favoráveis, de forma a entender as orientações e responder o questionário. Os critérios de exclusão foram: Profissionais afastados do serviço por motivos variados (férias, licenças e outros). Todos os participantes assinaram o Termo de Consentimento Livre e Esclarecido. A pesquisa obteve parecer favorável do Comitê de Ética em Pesquisa da Universidade Federal de São Paulo - UNIFESP, conforme Parecer n⿳0 56608.

Os dados foram coletados entre novembro de 2013 a fevereiro de 2014. Foi elaborado um plano de trabalho com informações sobre setores, data e horários agendados para coleta e sujeitos previamente sorteados para participarem da pesquisa. De posse destas informações os pesquisadores se dirigiam aos locais de coleta e se apresentavam a chefia responsável, informando sobre os procedimentos. Em seguida convidavam os participantes a responderem o questionário.

Uma vez coletados, os dados foram codificados e lançados em uma planilha eletrônica com dupla digitação e transferidos para um programa estatístico. Foram analisados com a técnica estatística multivariada de análise fatorial, cujo propósito é reduzir um conjunto de variáveis a outro de menor tamanho e mais controlável, permitindo sua compreensão ${ }^{(6)}$. A adequação da amostra foi mensurada pelos critérios de determinação da matriz de correlação, medida de adequação da amostra de KaiserMeyer-Olkin (KMO), teste de esfericidade de Bartlett e medida de consistência interna.

Os dados foram analisados em sequência: a) avaliação de dados por meio de análise fatorial exploratória (técnica R); b) correlação das variáveis pelo método de extração dos componentes principais; c) confirmação da confiabilidade e validade dos dados através dos testes de adequação da amostra e alfa de Cronbach; d) rotação dos fatores pelo método Varimax; e) análise da matriz de fatores, identificando, de modo hierárquico, os fatores de maior impacto sobre a humanização nos serviços de saúde.

\section{RESULTADOS}

Após a seleção dos participantes, foram aplicados 572 questionários, sendo 312 para profissionais de saúde, 211 para usuários e 49 para gestores. Na Tabela 1 a adequação da amostra pôde ser constatada, visto que a determinante da matriz de correlação obteve valores bastante elevados, indicando que as variáveis são consideravelmente correlacionadas, o que se confirma pelos testes de esfericidade de Bartlett e alfa de Cronbach. Tais valores rejeitam a hipótese nula de que não haja associação entre eles. Ao contrário, confirmam que existe associação estatisticamente significante, que indica ser a matriz de dados adequada à matriz fatorial.

Tabela 1 - Coeficiente de confiabilidade estatística da escala utilizada no estudo. Uberaba, MG, Brasil, 2014

\begin{tabular}{lccc} 
Indicador & \multicolumn{3}{c}{ Dimensão } \\
\cline { 2 - 4 } & Trabalho & Usuário & Gestão \\
\hline Alfa de Cronbach & 0,881 & 0,723 & 0,827 \\
\hline Medida de adequação da amostra de Kaiser-Meyer-Olkin & 0,884 & 0,684 & 0,722 \\
\hline Teste de esfericidade de Bartlett & 2188,148 & 1076,296 & 180,557 \\
\hline Qui-quadrado aprox. & & & \\
\hline Df & 325 & 171 & 55 \\
\hline Sig. & 0 & 0 & 0
\end{tabular}

Verifica-se na Tabela 2 a matriz fatorial na dimensão trabalho, que abarca sete fatores.

O fator 1, denominado "fator geral de adequação à $\mathrm{PNH}$ ", que corresponde ao tratamento unânime dispensado aos profissionais, implantação dos dispositivos da $\mathrm{PNH}$, acesso a um plano de carreira e recursos tecnológicos no trabalho, ambiente de trabalho seguro e profissionais identificados como sendo o fator mais importante para a humanização das condições de trabalho. 
Tabela 2 - Matriz fatorial na dimensão trabalho. Uberaba, MG, Brasil, 2014

\begin{tabular}{|c|c|c|}
\hline Fatores & Itens & Carga \\
\hline \multirow[t]{6}{*}{ 1. Fator geral de adequação à PNH } & Os profissionais são tratados da mesma maneira & 0,704 \\
\hline & A instituição prioriza a implantação de dispositivos da PNH & 0,679 \\
\hline & Recursos tecnológicos disponibilizados & 0,675 \\
\hline & Instituição oferece plano de carreira & 0,597 \\
\hline & Ambiente de trabalho seguro & 0,597 \\
\hline & Identificação dos profissionais & 0,522 \\
\hline \multirow{5}{*}{$\begin{array}{l}\text { 2. Satisfação com o trabalho e } \\
\text { reconhecimento profissional }\end{array}$} & Sentimento de realização com o trabalho & 0,714 \\
\hline & Satisfação com o salário recebido & 0,697 \\
\hline & Trabalho é reconhecido e valorizado & 0,645 \\
\hline & Satisfação com a gestão do serviço & 0,445 \\
\hline & Autonomia para executar o trabalho & 0,409 \\
\hline \multirow[t]{3}{*}{ 3. Comunicação e apoio da gestão } & Comunicação eficiente entre a equipe & 0,726 \\
\hline & Abertura para discutir sugestões & 0,705 \\
\hline & Comprometimento da gerência & 0,661 \\
\hline \multirow{3}{*}{$\begin{array}{l}\text { 4. Condições ambientais e recursos } \\
\text { materiais para o trabalho }\end{array}$} & Limpeza e higiene do local de trabalho & 0,75 \\
\hline & Sinalização do ambiente de trabalho & 0,717 \\
\hline & Recursos materiais para o trabalho & 0,535 \\
\hline \multirow{4}{*}{$\begin{array}{l}\text { 5. Conhecimento da área adstrita } \\
\text { da unidade e rede de atenção do } \\
\text { município }\end{array}$} & Número de trabalhadores na unidade & 0,657 \\
\hline & Integração entre os profissionais da equipe interdisciplinar & 0,57 \\
\hline & $\begin{array}{l}\text { Conhecimento da rede de atenção da área adstrita da } \\
\text { unidade e rede de atenção do município }\end{array}$ & 0,499 \\
\hline & Instituição desenvolve ações de qualidade de vida & 0,469 \\
\hline \multirow{4}{*}{$\begin{array}{l}\text { 6. Oportunidade de participação e } \\
\text { conhecimento do nível hierárquico }\end{array}$} & Conhecimento do organograma da instituição & 0,68 \\
\hline & Oportunidade de participar de atividades educativas & 0,572 \\
\hline & Conhecimento dos ocupantes da escala hierárquica & 0,539 \\
\hline & Oportunidade de fazer sugestões & 0,517 \\
\hline 7. Cobertura a outros setores & Cobertura a outros setores & 0,869 \\
\hline
\end{tabular}

Os profissionais de saúde participantes da pesquisa elegeram a questão da "satisfação com o trabalho e reconhecimento profissional" como o segundo fator mais importante. O terceiro fator agrupou as variáveis; comunicação eficiente entre a equipe, abertura para discutir sugestões e comprometimento da gerência; fator nomeado como "comunicação e apoio da gestão".

Observa-se ainda as variáveis incluídas no fator 4: a questão da higienização, sinalização da unidade e recursos materiais para o trabalho. Este fator foi intitulado como "condições ambientais e recursos materiais para o trabalho".

Em seguida há o número de trabalhadores e a integração destes na equipe multiprofissional em saúde, o conhecimento da área adstrita da unidade e rede de atenção do município como o quinto fator mais importante para a humanização das condições de trabalho.

Foi denominado "oportunidade de participação e conhecimento do nível hierárquico" o penúltimo fator na visão dos trabalhadores. Abrange as variáveis relacionadas ao conhecimento dos níveis hierárquicos, bem como seus ocupantes e oportunidade de participação das atividades educativas e de fazer sugestões. O último fator incluiu apenas a variável "cobertura a outros setores". A Tabela 3 aponta a matriz fatorial na dimensão dos usuários.

Na Tabela 3 verificamos que os usuários evidenciaram como mais importantes os aspectos preconizados na instituição: oferta de dispositivos, participação na gestão, oferta de atividades lúdicas e acesso aos serviços de ouvidoria. No segundo fator foram incluídas as variáveis relacionadas à resolutividade e confiança no serviço. Esses fatores foram denominados "visibilidade da PNH no serviço" e "resolutividade e confiança no serviço", respectivamente. 
Tabela 3 - Matriz fatorial na dimensão usuário. Uberaba, MG, Brasil, 2014

\begin{tabular}{|c|c|c|}
\hline Fatores & Itens & Carga \\
\hline \multirow[t]{4}{*}{ 1. Visibilidade da PNH no serviço } & Oferta de dispositivos da PNH & 0,818 \\
\hline & Participação na gestão & 0,816 \\
\hline & Oferta de atividades lúdicas & 0,706 \\
\hline & Acesso ao serviço de ouvidoria & 0,657 \\
\hline \multirow{3}{*}{$\begin{array}{l}\text { 2. Resolutividade e confiança no } \\
\text { serviço }\end{array}$} & Resolutividade & 0,873 \\
\hline & Atendimento rápido e resolutivo & 0,866 \\
\hline & Confiança no serviço & 0,51 \\
\hline \multirow[t]{2}{*}{ 3. Acolhimento } & Atendimento digno e respeitoso & 0,895 \\
\hline & Acolhimento; forma de recebimento na unidade & 0,892 \\
\hline \multirow[t]{4}{*}{ 4. Informações prestadas ao usuário } & $\begin{array}{lllll}\text { Informações sobre o funcionamento do serviço, } \\
\text { procedimentos e exames }\end{array}$ & 0,829 \\
\hline & Sinalização do ambiente/unidade & 0,557 \\
\hline & Informações adequadas e esclarecimento de dúvidas & 0,534 \\
\hline & Respeito às crenças do usuário & 0,366 \\
\hline \multirow[t]{2}{*}{ 5. Sigilo e confidencialidade } & Respeito à privacidade & 0,827 \\
\hline & Atendimento sigiloso e confidencial & 0,784 \\
\hline \multirow[t]{2}{*}{ 6. Ambiência } & Limpeza e higiene do serviço & 0,769 \\
\hline & Confortabilidade & 0,581 \\
\hline \multirow{2}{*}{$\begin{array}{l}\text { 7. Conhecimento e identificação dos } \\
\text { profissionais }\end{array}$} & Identificação dos profissionais & 0,566 \\
\hline & $\begin{array}{l}\text { Conhecimento da equipe responsável pelo atendimento/ } \\
\text { tratamento }\end{array}$ & 0,536 \\
\hline
\end{tabular}

Intitulado "acolhimento", o terceiro fator inclui as variáveis relacionadas à forma como o usuário é recebido e tratado na unidade e à prestação de um atendimento digno, respeitoso. No quarto fator estão as variáveis referentes a "informações prestadas ao usuário". A variável "respeito às crenças do usuário" esteve relacionada com este fator, embora com uma carga fatorial mais baixa.

No quinto, sexto e sétimo fatores estão incluídas as variáveis concernentes ao atendimento feito de maneira privativa, sigilosa e confidencial (fator 5), ambiência (fator 6) e identificação visual dos profissionais (fator 7). Apresenta-se na Tabela 4 a matriz fatorial na dimensão da gestão.

Tabela 4 - Matriz fatorial na dimensão gestão. Uberaba, MG, Brasil, 2014

\begin{tabular}{|c|c|c|}
\hline Fatores & Itens & Carga \\
\hline \multirow{7}{*}{$\begin{array}{l}\text { 1. Visibilidade dos princípios da PNH } \\
\text { no serviço }\end{array}$} & Atendimento acolhedor e resolutivo & 0,77 \\
\hline & $\begin{array}{l}\text { Processos de trabalho atendem aos requisitos da qualidade } \\
\text { em saúde }\end{array}$ & 0,762 \\
\hline & $\begin{array}{l}\text { Evidências objetivas de que as práticas de saúde são efetivas } \\
\text { e eficazes }\end{array}$ & 0,689 \\
\hline & $\begin{array}{l}\text { Usuários têm acesso a informações financeiras e tratamentos } \\
\text { realizados }\end{array}$ & 0,674 \\
\hline & $\begin{array}{l}\text { Prioridade das instâncias governamentais na implantação } \\
\text { da PNH }\end{array}$ & 0,602 \\
\hline & Horizontalização das linhas de mando & 0,565 \\
\hline & Política de redução das filas & 0,475 \\
\hline \multirow[t]{3}{*}{$\begin{array}{l}\text { 2. Impacto das diretrizes da PNH na } \\
\text { gestão do serviço }\end{array}$} & $\begin{array}{l}\text { Acesso a recursos financeiros para implantação dos } \\
\text { dispositivos da PNH }\end{array}$ & 0,882 \\
\hline & Gestão participativa com usuários e trabalhadores & 0,655 \\
\hline & Lógica da gestão privilegia a implantação da PNH & 0,515 \\
\hline 3. Acesso a serviços religiosos & Usuários têm acesso a serviços religiosos & 0,891 \\
\hline
\end{tabular}


Na Tabela 4 apresenta-se a atitude dos gestores em saúde acerca da influência da PNH nos serviços onde atuam. No fator 1 estão incluídas variáveis que representam o impacto de princípios elencados presentes nos serviços de saúde, como aspectos relacionados à forma de atendimento ao usuário, se o mesmo tem acesso a informações financeiras e tratamentos realizados. Aborda questões referentes à estruturação e gestão do serviço, como a efetividade das práticas, requisitos de qualidade e horizontalização das linhas de poder na instituição. Este fator foi nomeado como "visibilidade dos princípios da PNH no serviço".

Os gestores elegeram variáveis relacionadas ao acesso a recursos financeiros, participação dos usuários e trabalhadores e lógica de gestão voltada para a PNH como o segundo fator mais importante. De modo geral, pode-se afirmar que o "impacto das diretrizes da PNH na gestão do serviço" representa o segundo fator mais importante. A variável "usuários têm acesso a serviços religiosos" ficou isolada, no terceiro fator.

Encontrada uma solução fatorial confirmatória, buscou-se atribuir um significado aos fatores, mediante um esforço para nomear cada um deles. Variáveis com cargas fatoriais mais altas influenciaram a seleção do nome ou rótulo para representar cada fator.

\section{DISCUSSÃO}

A validade de construto refere-se ao grau em que um instrumento de medidas se relaciona consistentemente com outras medições assemelhadas, derivadas da mesma teoria e $\operatorname{conceitos}^{(5)}$. As técnicas de validação utilizadas nesta pesquisa ajudam a avaliar se as variáveis possuem significado científico e asseguram a exatidão necessária para as análises e conclusões a que se destinam.

A confiabilidade representa o grau em que uma variável é consistente com o que se pretende medir. Instrumentos que têm como propósito avaliar a humanização devem levar em conta as amplas situações que podem ser mensuradas nesse âmbito, dados objetivos, resultados concretos, mas também a transformação no próprio processo, na dinâmica dos serviços, na interação dos usuários ${ }^{(3)}$. $\mathrm{O}$ alfa de Cronbach foi adotado como medida de confiabilidade nesta pesquisa, sendo considerado consistente.

Nas Tabelas 2, 3 e 4 as categorias de fatores apareceram por ordem de variância nas três dimensões pesquisadas. O primeiro fator rotacionado nas três dimensões reflete as variáveis relacionadas ao grau de adequação às diretrizes da PNH e à visibilidade desta nos serviços de saúde. A gestão dos serviços de saúde desempenha um papel fundamental no quesito visibilidade, ao passo que a humanização só se torna realidade em uma instituição quando seus gestores a adotam enquanto um modelo de gestão. Ações pontuais não sustentam a humanização como processo transformador. "Os instrumentos que de fato asseguram esse processo são: a informação, a educação permanente, a qualidade e a gestão participativa"(7:257).

A percepção é um conceito amplo, diretamente relacionado às expectativas e experiências vivenciadas com a doença e o atendimento em saúde. No ambiente hospitalar, demonstrou ser um tema complexo, "com várias implicações que vão desde a própria compreensão do que venha a ser a humanização, até a operacionalização de algo que parece simples que às vezes não se efetiva, como uma boa comunicação entre o usuário, profissionais e gestores" (8:822). Desenvolver ações de humanização ancoradas na ética e respeito aos direitos dos profissionais de saúde contribui para uma melhor compreensão do tema.

Os profissionais de saúde elegeram a satisfação com o trabalho e o reconhecimento profissional como o segundo fator para a humanização do trabalho. A satisfação com o trabalho é resultante da complexa interação das "condições gerais de vida, das relações de trabalho, do processo de trabalho e do controle que os próprios trabalhadores possuem sobre suas condições de vida e trabalho" (9:77). Pode ser fonte de bem-estar, assim como causar prejuízos à "saúde física, mental e social, acarretando problemas à organização e ao ambiente de trabalho" ${ }^{\prime(9: 77)}$. Este ambiente desfavorável repercute nos aspectos relacionais entre profissionais e usuários, resultando em uma situação frágil para humanizar o cuidado ${ }^{(10)}$. 
A resolutividade e a confiança no serviço foram eleitas pelos usuários como o segundo fator mais importante. A primeira consiste na capacidade de resolução do sistema, sendo um dos requisitos das práticas humanizadas. É avaliada a partir dos resultados obtidos no atendimento dos usuários. Segundo o modelo hierarquizado de atenção à saúde, os serviços devem ser capazes de atender às demandas ou encaminhar os usuários para outros níveis de atenção(11). A confiança dos usuários no serviço de saúde melhora a aceitação do trabalho em equipe, com maior fidedignidade dos dados fornecidos e aumento da mobilização popular e luta por direitos e reivindicações ${ }^{(12)}$.

O impacto das diretrizes da PNH na gestão do serviço foi eleito como o segundo fator mais importante na visão dos gestores. O modelo de gestão clássica exerce forte influência na organização dos serviços de saúde, o que nem sempre garante que as práticas dos profissionais de saúde sejam eficazes. Como resultado, é observada uma desmotivação dos trabalhadores e baixa participação dos usuários ${ }^{(13)}$. Contrapondo-se ao modelo tradicional hegemônico, a PNH propõe a adoção de um modelo de gestão centrado no trabalho em equipe e na construção coletiva, que garantem que o poder seja de fato compartilhado ${ }^{(14)}$. As ações de humanização são apresentadas como diferencial competitivo e de qualidade, melhorando as práticas de gestão, ambiente físico, qualidade e resolutividade do atendimento, elevando a satisfação e o retorno financeiro da empresa ${ }^{(15)}$.

O terceiro fator na percepção dos profissionais de saúde se refere à questão da comunicação e apoio da chefia. O problema da comunicação vem ocupando as agendas da saúde, visto que entraves comprometem a qualidade do atendimento e mantêm o profissional refém de condições inadequadas, que não raro imputam desgaste e sofrimento psíquico ${ }^{(16)}$.

Na opinião dos usuários, o acolhimento foi eleito como o terceiro fator mais importante na humanização, sendo um desafio superar as dificuldades nas unidades de saúde. Mostrou-se como uma ferramenta "capaz de reduzir os níveis de insatisfação de usuários e profissionais, pois agiliza o serviço prestado ao cliente, reconhece prioridades e proporciona os devidos encaminhamentos para a continuidade de tratamento do usuário" ${ }^{\prime(17: 88)}$.

Na visão dos gestores, o acesso dos usuários a serviços religiosos representou o terceiro fator na dimensão gestão. Esta variável não se relacionou com as demais, mesmo com a utilização de técnicas fatoriais que aglutinam as variáveis em fatores. Interpretamos a posição dos gestores no seguinte aspecto: uma vez que a instituição se comprometa a ofertar serviços, deve também prover toda a estrutura necessária para tal, como área física, custos envolvidos com a manutenção desta e fluxo de pessoas dentro da instituição, o que impacta na segurança e no próprio controle de infecção hospitalar, por exemplo. Há ainda questões como a diversidade religiosa presente na população brasileira. Ressalta se que o acesso aos serviços religiosos é um fator relevante para humanização conforme a $\mathrm{PNH}^{(2)}$.

As condições ambientais de trabalho (limpeza, higienização e sinalização) e os recursos materiais para o trabalho foram aglutinados no quarto fator. "O problema em muitos locais é a falta de condições técnicas, seja de capacitação, seja de materiais, e como resultado o ambiente torna-se pouco humanizado pela má qualidade resultante e baixa resolutividade" (18:281).

Já para os usuários, o quarto fator está relacionado à questão das informações prestadas no serviço. Melhorias que elevem o nível da comunicação constituem uma das diretrizes centrais da $\mathrm{PNH}^{(2)}$. Por meio de uma comunicação eficiente se estabelece o debate e a divulgação de ideias que contribuem para a construção de projetos humanizantes, em sintonia com as políticas públicas voltadas à área de saúde.

Para os profissionais de saúde, o conhecimento da rede de atenção e área adstrita figurou como o quinto fator. Esta é representada por um espaço geográfico de onde provêm os indivíduos que estabelecem vínculo e relação com uma unidade de saúde. A rede de atenção à saúde responsabilizase pela integração e relacionamento entre os diferentes serviços. É uma rede heterogênea, "que se produz pelos entrelaçamentos que ocorrem entre diferentes atores, serviços, movimentos, políticas num dado território" (19:9). O fomento de redes no SUS tem sido o principal objetivo de todas as ações da $\mathrm{PNH}$.

Conforme os usuários, o quinto fator prima pela garantia do sigilo, confidencialidade e privacidade, que devem ser garantidos a todos os usuários, exceto em casos de interesse da coletividade. A privacidade refere-se "à proteção da intimidade do paciente, que muitas vezes pode ser garantida com 
uso de divisórias ou até mesmo com cortinas e elementos móveis"(20:11). Trata-se de medidas simples que resultam em "integração e privacidade, facilitando o processo de trabalho, aumentando a interação da equipe e ao mesmo tempo possibilitando atendimento personalizado" (20:11).

Segundo trabalhadores em saúde e usuários, a participação democrática e a ambiência figuraram como o sexto fator. A PNH ressalta a importância da gestão participativa nas unidades, promovendo a democratização das relações de trabalho( ${ }^{(2)}$. A ambiência "potencializa e facilita a capacidade de ação e reflexão das pessoas envolvidas nos processos de trabalho, possibilitando a produção de novas subjetividades" (20:12). Condições ambientais inadequadas geram desconforto e insatisfação por parte dos clientes internos e externos aos serviços de saúde.

A variável cobertura a outros setores foi eleita pelos trabalhadores como o último fator na dimensão trabalho. Acreditamos que esta situação é específica de algumas categorias profissionais, tais como Enfermagem, Nutrição e Agentes Comunitários de Saúde, não se estendendo às demais. Observase aqui a qualidade do método utilizado, sensível às variações entre os profissionais com diferentes realidades no ambiente de trabalho.

Os usuários elegeram a identificação dos profissionais e o conhecimento da equipe responsável pelo tratamento como o fator de menor relevância. O mais provável é que na visão do usuário, para ele identificar o profissional se torna de menor importância diante de tantos outros aspectos relevantes. Já para os profissionais os princípios da $\mathrm{PNH}$ recomendam tratar os usuários pelo nome e ter conhecimento da equipe de referência que os atenda ${ }^{(2)}$.

\section{CONCLUSÃO}

A análise fatorial exploratória indicou que o instrumento proposto atende aos requisitos psicométricos preconizados, apresentando características de consistência interna suficientes para avaliar a percepção de trabalhadores, gestores e usuários sobre aspectos da humanização nos serviços de saúde.

Observa-se a necessidade de novas etapas de validação em outros serviços de saúde e outras realidades em todos os níveis de atenção, permitindo a comparação dos resultados obtidos e a compreensão das prioridades dos segmentos trabalho, gestão e usuários que compõem os serviços de saúde em todos os níveis de atenção.

Os resultados da pesquisa são promissores, uma vez que indicam que o conjunto de variáveis selecionadas demonstrou ser capaz de mensurar o grau de humanização nos serviços de saúde, apresentando validade e confiabilidade para o que é proposto.

\section{REFERÊNCIAS}

1. Martins JT, Galdino MJQ, Garanhani ML, Sammi KM, Trevisan GS. Humanização no processo de trabalho na percepção dos Enfermeiros da Unidade de Terapia Intensiva. Cogitare Enferm. [Internet] 2015;20(3) [acesso em 22 fev 2016]. Disponível: http://dx.doi.org/10.5380/ce.v20i3.41521.

2. Ministério da Saúde (BR). Secretaria de Atenção à Saúde. Núcleo Técnico da Política Nacional de Humanização. HumanizaSUS: documento base para gestores e trabalhadores do SUS. Série B. Textos Básicos de Saúde, Brasília: Ministério da Saúde; 2006.

3. Santos-Filho SB. Perspectivas da avaliação na Política Nacional de Humanização em Saúde: aspectos conceituais e metodológicos. Ciênc. saúde coletiva. [Internet] 2007;12(4) [acesso em 06 jun 2014]. Disponível: http://dx.doi. org/10.1590/S1413-81232007000400021.

4. de Oliveira D, Walter SA, Bach TM. Critérios de validade em pesquisas em estratégia: uma análise em artigos publicados no EnAnpad de 1997 a 2010. RAM, Rev. Adm. Mackenzie. [Internet] 2012;13(6) [acesso em 06 jun 2014]. Disponível: http://dx.doi.org/10.1590/S1678-69712012000600010.

5. Martins GA. Sobre Confiabilidade e Validade. Revista Brasileira de Gestão de Negócios. 2006;8(20):1-12. 
6. de Moraes OB, Abiko AK. Utilização da análise fatorial para a identificação de estruturas de interdependência de variáveis em estudos de avaliação pós-ocupação. XI Encontro Nacional de Tecnologia do Ambiente Construído. [Internet] 2006 [acesso em 06 jun 2014]. Disponível: http://www.infohab.org.br/entac2014/2006/ artigos/ENTAC2006_1233_1242.pdf.

7. Rios IC. Humanização: a essência da ação técnica e ética nas práticas de saúde. Rev. bras. educ. med. 2009;33(2):253-61.

8. Garcia AV, Argenta CE, Sanchez KR, de São Thiago ML. O grupo de trabalho de humanização e a humanização da assistência hospitalar: percepção de usuários, profissionais e gestores. Physis. [Internet] 2010;20(3) [acesso em 06 jun 2014]. Disponível: http://dx.doi.org/10.1590/S0103-73312010000300007.

9. Marqueze EC, Moreno CRC. Satisfação no trabalho - uma breve revisão. Rev. bras. saúde ocup. [Internet] 2005;30(112) [acesso em 07 jul 2014]. Disponível: http://dx.doi.org/10.1590/S0303-76572005000200007.

10. Fontana RT. Humanização no processo de trabalho em enfermagem: uma reflexão. Rev. Rene. 2010;11(1):2007.

11. Turrini RNT, Lebrão ML, Cesar CLG. Resolutividade dos serviços de saúde por inquérito domiciliar: percepção do usuário. Cad. Saúde Pública. 2008;24(3):663-74.

12. Valentim IVL, Kruel AJ. A importância da confiança interpessoal para a consolidação do Programa de Saúde da Família. Ciênc. saúde coletiva. 2007;12(3):777-88.

13. Alves CA, Deslandes SF, Mitre RMA. A gestão do processo de trabalho da enfermagem em uma enfermaria pediátrica de média e alta complexidade: uma discussão sobre cogestão e humanização. Interface. [Internet] 2011;15(37) [acesso em 05 jun 2014]. Disponível: http://dx.doi.org/10.1590/S1414-32832011005000008.

14. Ministério da Saúde (BR). Secretaria de Atenção à Saúde. Política Nacional de Humanização da Atenção e Gestão do SUS. Gestão participativa e cogestão. Brasília: Ministério da Saúde; 2009.

15. Backes DS, Silva DM, de Siqueira HH, Erdmann AL. O produto do serviço de enfermagem na perspectiva da gerência da qualidade. Rev. Gaúcha Enferm. 2007;28(2):163-70.

16. Deslandes SF. Análise do discurso oficial sobre a humanização da assistência hospitalar. Ciênc. saúde coletiva. 2004;9(1):7-14.

17. do Nascimento ERP, Hilsendeger BR, Neth C, Belaver GM, Bertoncello KCG. Classificação de risco na emergência: avaliação da equipe de enfermagem. Rev. enferm. UERJ. [Internet] 2011;19(1) [acesso em 05 jun 2014]. Disponível: http://www.facenf.uerj.br/v19n1/v19n1a14.pdf.

18. de Oliveira BRG, Collet N, Viera CS. A humanização na assistência à saúde. Rev. Latino-Am. Enfermagem. [Internet] 2006;14(2) [acesso em 20 ago 2014]. Disponível: http://dx.doi.org/10.1590/S0104-11692006000200019.

19. Ministério da Saúde (BR). Secretaria de Atenção à Saúde. Política Nacional de Humanização da Atenção e Gestão do SUS. Redes de produção de saúde. Série B. Textos Básicos de Saúde, Brasília (DF): Ministério da Saúde; 2009.

20. Ministério da Saúde (BR). Secretaria de Atenção à Saúde. Núcleo Técnico da Política Nacional de Humanização. Ambiência. Série B. Textos Básicos de Saúde, Brasília (DF): Ministério da Saúde; 2010. 